\title{
Fluxes and Chern Morphisms of Hyperbolic Orbifolds
}

\author{
A. A. Bytsenko \\ Departamento de Física, Universidade Estadual de Londrina, Caixa Postal 6001, Londrina, PR, Brazil \\ and M. E. X. Guimarães \\ Departamento de Matemática, Universidade de Brasília, CEP: 70910-900, Brasília, DF, Brazil \\ (Received on 11 October, 2005)
}

\begin{abstract}
Methods of $K$-theory and spectral theory of Dirac operators are applied to describe the Chern isomorphisms and quantum fields on branes on hyperbolic manifolds.
\end{abstract}

\section{INTRODUCTION}

In superstring and superconformal field theories $D$-branes play a significant role. The $K$-theory method has been applied to compute $D$-brane charges [1-3] which identifies with elements of Grothendick $K$-groups [4-6] for horizon manifolds. The relevant description of the Ramond-Ramond charges in term of equivariant $K$-theory has been demonstrated in [1, 7-9]. It leads to a formalism of fractional branes pinned on the orbifold singularities which have components in the twisted sectors of the closed strings.

In this note the Chern isomorphism for fluxes and brane charges interpretation is considered. We examine the Chern classes and charges of branes using the methods of $K$-theory and spectral theory of differential operators related to hyperbolic spaces (for string compactifications with hyperbolic horizons, see for example [10]).

\section{RAMOND-RAMOND FIELDS OVER BRANE}

The Ramond-Ramond fields and their flux quantization could be formulated in terms of differential $K$-theory [11, 12]. These fields get precise characterization by means of bundles with connections [13]. The groups $K^{j}(X, U(1))$ (see $[14]$ for details) describe the flat Ramond-Ramond fluxes on a compactification $X$, where $j$ matters modulo two by Bott periodicity and $j=1$ for the IIA and $j=0$ for the IIB theory. These groups fit the following exact sequence:



The maps from $K$-theory to cohomology are the usual Chern character maps, that are isomorphisms over the reals. Since the character is one of the features of the $K$-theory, which is useful in a variety of applications, we shall review briefly the Chern construction in the topological $K$-theory.

For a finite-dimensional smooth manifold $X$ the RamondRamond phase admits a description of the form [15]:

$$
0 \rightarrow \frac{H^{\text {odd }}(X, \mathbb{R})}{K^{1}(X) / \text { Tor }} \rightarrow K^{1}(X, U(1)) \rightarrow \operatorname{Tor} K^{0}(X) \rightarrow 0
$$

The component group is given by the torsion classes in $K^{0}(X)$, while the trivial component consists of the torus $H^{\text {odd }}(X, \mathbb{R}) /\left(K^{1}(X) /\right.$ Tor $)$. The interpretation of the elements of the group $K^{1}(X, U(1))$ is complicate, we can restrict ourselves to the case where the cohomology $H^{\text {odd }}(X, \mathbb{R})$ vanishes. The group of fluxes is simply $K^{1}(X, U(1)) \cong \operatorname{Tor} K^{0}(X)$, and the corresponding Ramond-Ramond flux can be represented by a pair $\left(E_{\chi}, E_{\chi^{\prime}}\right)$ of flat vector bundles with $\operatorname{rank}\left(E_{\chi}\right)=$ $\operatorname{rank}\left(E_{\chi^{\prime}}\right)$, i.e., a virtual flat bundle $\triangle E_{\chi}=E_{\chi}-E_{\chi^{\prime}}$ of rank zero (see Section 3 for notations). In the string theory path integral a torsion Ramond-Ramond flux gives an additional phase factor to a $D$-brane. A brane can be represented by a $K$-homology class, that is a map of an odd spin-manifold $X$ (possibly equipped with an additional Chan-Paton vector bundle) into the space-time $M$ [15]. Therefore, the holonomy of the Ramond-Ramond fields over this brane can be associated with the eta invariant of virtual bundle $\triangle E_{\chi}$ restricted to $X$. The complex eta functions of hyperbolic spaces will be considered in the next section.

\section{CHERN CLASSES AND $K$-THEORY OF HYPERBOLIC ORBIFOLDS}

\section{A. $U(n)$-gauge bundles and the Chern-Simons invariants}

Methods of spectral theory of differential operators can be used in the type II string compactified on spaces with the real hyperbolic horizon $X$. Let $X=G / \mathcal{K}$ be an irreducible rank one symmetric space of non-compact type. Thus $G$ will be a connected non-compact simple split rank one Lie group with finite center, and $\mathcal{K} \subset G$ will be a maximal compact subgroup. The object of interest is the groups $G=S O_{1}(n, 1)\left(n \in \mathbb{Z}_{+}\right)$and $\mathcal{K}=S O(n)$. The corresponding symmetric space of non-compact type is the real hyperbolic space $X=\mathbb{H}^{n}=S O_{1}(n, 1) / S O(n)$ of sectional curvature -1 . Let $X_{\Gamma}=\Gamma \backslash G / \mathcal{K}$ be a real compact hyperbolic manifold. The fundamental group of $X_{\Gamma}$ acts by covering transformations on $X$ and gives rise to a discrete, co-compact subgroup $\Gamma \subset G$. Let $P=X_{\Gamma} \otimes \mathfrak{G}$ be a principal bundle over $X_{\Gamma}$ with the gauge group $\mathfrak{G}=U(n)$ and let $U_{X_{\Gamma}}=\Omega^{1}\left(X_{\Gamma} ; \mathfrak{g}\right)$ be the space of all connections on $P$; this space is an affine space of one-forms on $X_{\Gamma}$ with values in the Lie algebra $\mathfrak{g}$ of $\mathfrak{G}$. Suppose that $\chi$ is any one-dimensional representation of $\Gamma$ factors through a representation of $H^{1}(X ; \mathbb{Z})$. It can be shown that for a unitary representation $\chi: \Gamma \rightarrow U(n)$, the corresponding flat vector 
bundle $\mathbb{E}_{\chi}$ is topologically trivial $\left(\mathbb{E}_{\chi} \cong X \otimes \mathbb{C}^{n}\right)$ if and only if $\left.\operatorname{det} \chi\right|_{\text {Tor }^{1}}:$ Tor $^{1} \rightarrow U(1)$ is the trivial representation. Here Tor ${ }^{1}$ is the torsion part of $H^{1}(M ; \mathbb{Z})$ and $\operatorname{det} \chi$ is a one-dimensional representation of $\Gamma \operatorname{defined}$ by $\operatorname{det} \chi(\gamma):=\operatorname{det}(\chi(\gamma))$, for $\gamma \in \Gamma$.

A three form flux associates a phase to a Euclidean brane world-volume $X_{\Gamma}$ which is given by the eta invariant of the virtual bundle $\eta_{E_{\chi}}-\eta_{E^{\prime}}$, restricted to $X_{\Gamma}$. We can express this phase directly in terms of the Chern-Simons invariant. For any extension $\widetilde{A}_{\chi}$ of a flat connection $A_{\chi}$ corresponding to $\chi$ the second Chern character $\operatorname{ch}_{2}\left(\widetilde{\mathbb{E}}_{\chi}\right)\left(=-\left(1 / 8 \pi^{2}\right) \operatorname{Tr}\left(F_{\widetilde{A}_{\chi}} \wedge F_{\widetilde{A}_{\chi}}\right)\right)$ of $\widetilde{\mathbb{E}}_{\chi}$ can be expressed in terms of the first and second Chern classes: $\operatorname{ch}_{2}\left(\widetilde{\mathbb{E}}_{\chi}\right)=(1 / 2) c_{1}\left(\widetilde{\mathbb{E}}_{\chi}\right)^{2}-c_{2}\left(\widetilde{\mathbb{E}}_{\chi}\right)$. The Chern character and the $\widehat{A}$-genus, the usual polynomial related to Riemannian curvature $\Omega$, are given by

$$
\begin{aligned}
\operatorname{ch}\left(\widetilde{\mathbb{E}}_{\chi}\right) & =\operatorname{rank} \widetilde{\mathbb{E}}_{\chi}+c_{1}\left(\widetilde{\mathbb{E}}_{\chi}\right)+\operatorname{ch}_{2}\left(\widetilde{\mathbb{E}}_{\chi}\right) \\
& =\operatorname{dim} \chi+c_{1}\left(\widetilde{\mathbb{E}}_{\chi}\right)+\operatorname{ch}_{2}\left(\widetilde{\mathbb{E}}_{\chi}\right), \\
\widehat{A}\left(\Omega^{M}\right) & =1-\frac{1}{24} p_{1}\left(\Omega^{M}\right) .
\end{aligned}
$$

Here $p_{1}\left(\Omega^{M}\right)$ is the first Pontriagin class, $\Omega^{M}$ is the Riemannian curvature of four-manifold $M$ which has a boundary $\partial M=X_{\Gamma}$. Thus we have

$$
\begin{aligned}
\operatorname{ch}\left(\widetilde{\mathbb{E}}_{\chi}\right) \widehat{A}\left(\Omega^{M}\right)= & \left(\operatorname{dim} \chi+c_{1}\left(\widetilde{\mathbb{E}}_{\chi}\right)+\operatorname{ch}_{2}\left(\widetilde{\mathbb{E}}_{\chi}\right)\right) \\
& \times\left(1-\frac{1}{24} p_{1}\left(\Omega^{M}\right)\right) \\
= & \operatorname{dim} \chi+c_{1}\left(\widetilde{\mathbb{E}}_{\chi}\right)+\operatorname{ch}_{2}\left(\widetilde{\mathbb{E}}_{\chi}\right) \\
- & \frac{\operatorname{dim} \chi}{24} p_{1}\left(\Omega^{M}\right) .
\end{aligned}
$$

The integral over the manifold $M$ takes the form

$$
\int_{M} \operatorname{ch}\left(\widetilde{\mathbb{E}}_{\chi}\right) \widehat{A}\left(\Omega^{M}\right)=\int_{M} \operatorname{ch}_{2}\left(\widetilde{\mathbb{E}}_{\chi}\right)-\frac{\operatorname{dim} \chi}{24} \int_{M} p_{1}\left(\Omega^{M}\right) .
$$

For any representation $\chi$ one can construct a vector bundle $\widetilde{\mathbb{E}}_{\chi}$ over a certain four-manifold $M$ which is an extension of a flat complex vector bundle $\mathbb{E}_{\chi}$ over $X_{\Gamma}$. The second Chern character for the $U(1)$ part is half the Chern-Simons term. The relevant cobordism group vanish, and in fact for three-manifold $X_{\Gamma}$ we found a four-manifold $M$ and extended the $U(n)$ bundle such that manifold $M$ is spin. Therefore the intersection form on $M$ allows one to define the Chern-Simons invariant using $c_{1}^{2}$. For the chern classes we have $c_{1}\left(\mathbb{E}_{\chi}\right) \in H^{2}\left(X_{\Gamma}, \mathbb{Z}\right)$, $c_{2}\left(\mathbb{E}_{\chi}\right) \in H^{4}\left(X_{\Gamma}, \mathbb{Z}\right)$, and $H^{4}\left(X_{\Gamma}, \mathbb{Z}\right) \cong \mathbb{Z}_{|\Gamma|}$.

The Dirac index is given by [16-18]

$$
\begin{aligned}
\operatorname{Index} D_{\widetilde{A}_{\chi}} & =\int_{M} \operatorname{ch}\left(\widetilde{\mathbb{E}}_{\chi}\right) \widehat{A}(M) \\
& -\frac{1}{2}\left(\eta\left(0, \mathfrak{D}_{\chi}\right)+h\left(0, \mathfrak{D}_{\chi}\right)\right),
\end{aligned}
$$

where $h\left(0, \mathfrak{D}_{\chi}\right)$ is the dimension of the space of harmonic spinors on $X_{\Gamma}\left(h\left(0, \mathfrak{D}_{\chi}\right)=\operatorname{dimKer} \mathfrak{D}_{\chi}=\right.$ multiplicity of the 0 -eigenvalue of $\mathfrak{D}_{\chi}$ acting on $\left.X_{\Gamma}\right) ; \mathfrak{D}_{\chi}$ is a Dirac operator on $X_{\Gamma}$ acting on spinors with coefficients in $\chi$. The ChernSimons action $C S_{U(n)}\left(\widetilde{A}_{\chi}\right)=-\left(1 / 8 \pi^{2}\right) \int_{M} \operatorname{Tr}\left(F_{\widetilde{A}_{\chi}} \wedge F_{\widetilde{A}_{\chi}}\right)$ can be derived from Eq. (5). Indeed,

$$
\begin{aligned}
\operatorname{Index} D_{\widetilde{A}_{\chi}} & =C S_{U(n)}\left(\widetilde{A}_{\chi}\right)-\frac{\operatorname{dim} \chi}{24} \int_{M} p_{1}\left(\Omega^{M}\right) \\
& -\frac{1}{2}\left(\eta\left(0, \mathfrak{D}_{\chi}\right)+h\left(0, \mathfrak{D}_{\chi}\right)\right) .
\end{aligned}
$$

There exists a Selberg type (Shintani) zeta function $Z\left(s, \mathfrak{D}_{\chi}\right)$ associated with a twisted Dirac operator $\mathfrak{D}_{\chi}$ acting on oriented odd dimensional real hyperbolic spaces. $Z\left(s, \mathfrak{D}_{\chi}\right)$ is a meromorphic on $\mathbb{C}$, and for $\mathfrak{R}\left(s^{2}\right) \gg 0$ one has [19]:

$$
\log Z\left(0, \mathfrak{D}_{\chi}\right)=\sqrt{-1} \pi \eta\left(0, \mathfrak{D}_{\chi}\right) .
$$

Thus, finally we get the $U(n)$-Chern-Simons invariant of an irreducible flat connection on the real hyperbolic threemanifolds:

$$
\begin{aligned}
C S_{U(n)}\left(\widetilde{A}_{\chi}\right) & -\operatorname{modulo}(\mathbb{Z} / 2) \\
& =\frac{1}{2}\left(\operatorname{dim} \chi \eta(0, \mathfrak{D})-\eta\left(0, \mathfrak{D}_{\chi}\right)\right) \\
& =\frac{1}{2 \pi \sqrt{-1}} \log \left[\frac{Z(0, \mathfrak{D})^{\operatorname{dim} \chi}}{Z\left(0, \mathfrak{D}_{\chi}\right)}\right] .
\end{aligned}
$$

The value of the Chern-Simons functional on the space of connections at a critical point can be regarded as a topological invariant of a pair $\left(X_{\Gamma}, \chi\right)$. If the one form flux is zero and $c_{1}\left(\mathbb{E}_{\chi}\right)=0$ then the flux is measured by $c_{2}\left(\mathbb{E}_{\chi}\right) \in$ $H^{3}\left(X_{\Gamma}, U(1)\right)$. This group of three-form fluxes is generated by the two-dimensional representation and is given by [15]: $H^{4}\left(X_{\Gamma}, \mathbb{Z}\right)=H^{3}\left(X_{\Gamma}, U(1)\right)=\mathbb{Z}_{|\Gamma|}$. Using $K$-theory the groups of fluxes can be computed confirming our computation via the Chern-Simons invariants (for more details see [15]).

\section{B. Brane charges}

Before discussing the brane charge formula we begin with some conventions which apply throughout. Let $X$ be an oriented manifold, and let $H^{*}(X)$ be the cohomology ring of $X$. The Poincaré duality, which is well-known result of differential topology, gives a canonical isomorphism

$$
\begin{aligned}
\mathfrak{d}_{X}: & H^{j}(X) \stackrel{\approx}{\longrightarrow} H_{j-p}(X), \\
& \text { for all } p=0,1, \ldots, n=\operatorname{dim} X
\end{aligned}
$$

Let $f: Y \rightarrow X$ be a continuous map from $Y$ to $X$ and $m=$ $\operatorname{dim} Y$. For all $p \geq m-n$ there is a linear map, called the Gysin homomorphism:

$$
f_{!}: H^{j}(Y) \longrightarrow H^{j-(m-n)}(X)
$$

which is defined such a way that the sequence

$$
\begin{aligned}
& H^{p}(Y) \stackrel{\mathfrak{d}_{Y}}{\longrightarrow} H_{m-p}(Y) \stackrel{f_{*}}{\longrightarrow} H_{m-p}(X) \\
& H_{m-p}(X) \stackrel{\mathfrak{d}_{X}^{-1}}{\longrightarrow} H^{p-(m-n)}(X) H^{p-(m-n)}(X) \stackrel{f_{!}}{\longleftarrow} H^{p}(Y)
\end{aligned}
$$


is commutative. Thus $f_{!}=\mathfrak{d}_{X}^{-1} f_{*} \mathfrak{d}_{Y}$, where $f_{*}$ is the natural push-forward map acting on homology. As an example of that construction let us assume that $Y$ is an oriented vector bundle $E$ over $X$ of fiber dimension $\ell$. The canonical projection map $\pi: E \rightarrow X$ and the inclusion $i: X \rightarrow E$ of the zero section induce maps on homology with $\pi_{*} i_{*}=$ Id. For all $j$ we have the following isomorphisms:

$$
\pi_{!}: H^{j+\ell}(E) \stackrel{\approx}{\longrightarrow} H^{j}(X) \quad i_{!}: H^{j}(X) \stackrel{\approx}{\longrightarrow} H^{j+\ell}(E)
$$

$\pi_{!}$is the Gysin map; it can be associated with integration over the fibers of $E \rightarrow X$. We have $\pi_{!} i_{!}=\mathrm{Id}$, so that $\pi_{!}=\left(i_{!}\right)^{-1}$. The map $i_{\text {! }}$ is called the Thom isomorphism of the oriented vector bundle $E$. The particular example $j=0$ is an important case of the Thom isomorphism. For $j=0$ a map $H^{0}(X) \rightarrow$ $H^{\ell}(E)$, and the image of $1 \in H^{0}(X)$ determines a cohomology class $\Phi[E]=i_{!}(1) \in H^{\ell}(E)$, which is called the Thom class of $E$.

Let us consider $U(n)$ gauge bundle $\mathbb{E}$ on the brane. It has been shown that, as an element of $H^{*}(X)$, the RamondRamond charge associated with a $D$-brane wrapping a supersymmetric cycle in spacetime $f: Y \hookrightarrow X$ with Chan-Paton bundle $\mathbb{E} \rightarrow Y$ is given by

$$
Q=\operatorname{ch}\left(f_{!} \mathbb{E}\right) \wedge[\widehat{A}(T X)]^{1 / 2} .
$$

The map

$$
\operatorname{ch}: K(X) \otimes_{\mathbb{Z}} \mathbb{Q} \longrightarrow H^{\text {even }}(X, \mathbb{Q}) \equiv \bigoplus_{n \geq 0} H^{2 n}(X, \mathbb{Q})
$$

is an isomorphism, and it can be extend to a ring isomorphism [20]: ch: $K^{*}(X) \otimes_{\mathbb{Z}} \mathbb{Q} \stackrel{\approx}{\longrightarrow} H^{*}(X, \mathbb{Q})$, which maps $K^{-1}(X) \otimes_{\mathbb{Z}}$ $\mathbb{Q}$ onto $H^{\text {odd }}(X, \mathbb{Q})$. Note that the rational cohomology ring $H^{\text {even }}(X, \mathbb{Q})$ has a natural inner product, while the pairing $K(X)$, associated with the cohomology ring $K(X) \otimes_{\mathbb{Z}} \mathbb{Q}$, is given by the index of the Dirac operator.

The result (9) is in complete agreement with the fact that $D$-brane charge is given by $f_{!}[\mathbb{E}] \in K(X)$, and it gives an explicit formula for the brane charges in terms of the Chern character homomorphism on $K$-theory. The Chern characters $\mathrm{ch}^{*}$ (cohomology) and $\mathrm{ch}_{*}$ (homology) preserve the "cap" product $\cap$. It means that for every it topological space $X$ there is a $\mathbb{Z}_{2}$ - degree preserving commutative sequence [21, 22]:

$$
\begin{aligned}
& K^{*}(X) \bigotimes K_{*}(X) \stackrel{\cap}{\longrightarrow} K_{*}(X) \stackrel{\mathrm{ch}_{*}}{\longrightarrow} H_{*}(X, \mathbb{Q}) \\
& H_{*}(X, \mathbb{Q}) \stackrel{\cap}{\longleftarrow} H^{*}(X, \mathbb{Q}) \bigotimes H_{*}(X, \mathbb{Q}) \\
& H^{*}(X, \mathbb{Q}) \bigotimes H_{*}(X, \mathbb{Q}) \stackrel{\mathrm{ch}^{*} \otimes \mathrm{ch}_{*}}{\longleftarrow} K^{*}(X) \bigotimes K_{*}(X)
\end{aligned}
$$

For a finite $\mathrm{CW}$-complex $X, K_{*}(X)$ is a finitely generated abelian group and $\mathrm{ch}_{*}$ induced an isomorphism $K_{*}(X) \otimes_{\mathbb{Z}}$ $\mathbb{Q} \longrightarrow H_{*}(X, \mathbb{Q})$ of $\mathbb{Z}_{2}-$ graded vector spaces over $\mathbb{Q}$.

\section{Spaces stratified fibered over hyperbolic orbifolds}

Here we consider algebraic $K$-groups which can be applied for computation of charges of branes located at points of spaces stratified fibered over real hyperbolic spaces. Let as before $X_{\Gamma}$ be a closed (means compact and without boundary) connected Riemannian manifold with negative curvature. Let $Y^{1} \subset Y^{2} \subset Y^{3} \subset \ldots$ be a sequence of connected compact smooth manifolds and $F$ be a finite group which acting on $X_{\Gamma}$ via isometries and on each $Y^{\ell}$ via smooth maps. Assume also the smooth embedding $Y^{\ell} \subset Y^{\ell+1}$ is both $F$-equivariant and $\ell$-connected. Let $Y \equiv Y^{\infty}=\cup_{\ell=1}^{\infty} Y^{\ell}$ and give $Y$ the direct limit topology. The induced action of $F$ on $Y$ is free. Assuming that $\left\{W^{\ell}\right\}_{\ell=1}^{\infty}$ is the orbit space $X_{\Gamma} \times Y^{\ell}$ under the diagonal action of $F$ and $W \equiv W^{\infty}$ is direct limit $_{\ell \rightarrow \infty} W^{\ell}$. Let $X$ be the orbit space $X_{\Gamma} / F$ and $\rho_{\ell}: W^{\ell} \rightarrow X$ be the map induced from the canonical projection of $X_{\Gamma} \times Y^{\ell}$. The each $\rho_{\ell}(\ell=1,2, \ldots, \infty)$ is a stratified system of fibrations on $X$ [23] (Definition 8.2).

Let $R$ be a ring with unity and $G L_{n}(R)$ be the invertible $n \times n$ matrices with entries in $R$. Let $E_{n}(R)$ be the subgroup of elementary matrices. By definition an elementary matrix $E_{i j}(a)$ has 1 on the diagonal entry, $a \in R-\{0\}$ at the $(i, j)$-position while the remaining entries are 0 . Define $G L:=\lim _{n \rightarrow \infty} G L_{n}(R), E(R):=\lim _{n \rightarrow \infty} E_{n}(R)$; the limit is taken over the following maps: $G L_{n}(R) \rightarrow G L_{n+1}(R),(a) \mapsto$ $\operatorname{diag}(a, 1)$. Let

$$
C_{*}:=C_{0} \longrightarrow C_{1} \longrightarrow \ldots \longrightarrow C_{\ell}
$$

be a finite $C W$-complex.

The Whitehead group $W h(\Gamma)$ of $\Gamma$ is $C_{1}(\mathbb{Z}[\Gamma]) / \sigma(<$ $\Gamma,-\Gamma>)$, where $C_{1}(\mathbb{Z}[\Gamma])=G L(\mathbb{Z}[\Gamma]) / E(\mathbb{Z}[\Gamma])$, and $\sigma$ : $\left\langle\Gamma,-\Gamma>\rightarrow K_{1}(\mathbb{Z}[\Gamma])\right.$ is determine by sending $\pm \gamma$ to the $1 \times 1$ matrix $( \pm \gamma), \forall \gamma \in \Gamma$. As a consequence, $W h(1)=0$. The group $W h(\Gamma)$ has been checked for several classes of groups: for free Abelian groups [24]; for free non-Abelian groups [25]; for the fundamental group of any complete nonpositively curved Riemannian manifold [26]; for the fundamental group of finite polyhedra with non-positive curvature [27]; for the fundamental group of any Haken three-manifold [28], etc. The Whitehead group of the finite cyclic group is trivial [29].

Let $Y$ be a contractible; denoting $\pi_{1} W$ by $\Gamma$ we have result valid all integers $n$ [30]:

$$
\begin{aligned}
W h_{n}(\Gamma) \otimes \mathbb{Q} & \simeq \bigoplus_{\ell=0}^{\infty} H_{\ell}\left(X ; W h_{n-\ell}\left(G_{y}\right) \otimes \mathbb{Q}\right), \\
K_{n}(\mathbb{Z} \Gamma) \otimes \mathbb{Q} & \simeq \bigoplus_{\ell=0}^{\infty} H_{\ell}\left(X ; K_{n-\ell}\left(\mathbb{Z} G_{y}\right) \otimes \mathbb{Q}\right) .
\end{aligned}
$$

Here $G_{y}=\pi_{1}\left(\rho^{-1}(y)\right)$ for $y \in X$ and $W h_{n-\ell}\left(G_{y}\right) \otimes \mathbb{Q}$, $K_{n-\ell}\left(\mathbb{Z} G_{y}\right) \otimes \mathbb{Q}$ are the corresponding stratified systems of Abelian groups over $X$. More precise information contains the following formulas [30]:

$$
K_{n}(\mathbb{Z} \Gamma) \otimes \mathbb{Q} \cong \bigoplus_{\ell=0}^{\infty} H_{\ell}\left(\Gamma \backslash G / \mathcal{K} ; \mathfrak{K}_{n-\ell}\right),
$$

$\mathfrak{K}_{\ell}(\Gamma \backslash \mathfrak{g} / \mathcal{K})$ is isomorphic to $K_{\ell}\left(\mathbb{Z}\left(\Gamma \bigcap \mathfrak{g} \mathcal{K} \mathfrak{g}^{-1}\right)\right) \otimes \mathbb{Q}$,

where $\mathfrak{K}_{\ell}$ is a stratified system of $\mathbb{Q}$ vector spaces over $\Gamma \backslash G / \mathcal{K}$, and $\Gamma \cap \mathfrak{g} \mathcal{K} \mathfrak{g}^{-1}$ is a finite subgroup of $\Gamma$. 
The isomorphism conjecture of Farrell and Jones [31] states that the algebraic $K$-theory of the integral group ring $\mathbb{Z} \Gamma$ may be computed from the algebraic $K$ - theory of the virtually cyclic subgroups of $\Gamma$ (a group $\Gamma$ is called virtually cyclic if it has a cyclic subgroup of finite index). For precise statement and definitions see [32]. In [31] the isomorphism conjecture has been proved in lower algebraic $K$-theory for co-compact discrete subgroups of a virtually connected Lie group, in particular for discrete groups acting discontinuously and co-compactly by isometries on a simply connected Riemannian manifold $X$ with non-positive sectional curvature. In
[33] this result has been extended to the discrete groups acting discontinuously on real hyperbolic $n$-space via isometries whose orbit space has finite volume.

\section{Acknowledgements}

The authors would like to thank $\mathrm{CNPq}$ and Fundação Araucária (Paraná) for support.
[1] E. Witten, D-branes and $K$-theory, JHEP 9812, 019 (1998) [hep-th/9810188].

[2] S. Gukov, K-Theory, Relality, and Orientifolds, Commun. Math. Phys. 210, 621 (2000) [hep-th/9901042].

[3] E. Sharpe, D-Branes, Derived Categories, and Grothendieck Groups, Nucl. Phys. B 561, 433 (1999) [hep-th/9902116].

[4] M. F. Atiyah, $K-$ Theory, Benjamin, New York, 1967.

[5] M. Karoubi, K-Theory. An Introduction, Springer-Verlag, Berlin 1978.

[6] D. Husemoller, Fibre Bundles. McGraw-Hill, New York 1966.

[7] D. Diaconescu and J. Gomis, Fractional branes and boundary states in orbifold theories, JHEP 0010, 001 (2000) [hepth/9906242].

[8] H. Garcia-Compean, D-branes in orbifold singularities and equivariant $K$-theory, Nucl. Phys. B 557, 480 (1999) [hepth/9812226].

[9] O. Bergman, E. Gimon, and B. Kol, Strings on Orbifold Lines, JHEP 0105, 019 (2001) [hep-th/0102095].

[10] A. A. Bytsenko, M. E. X. Guimarães, and J. A. Helayel-Neto, Hyperbolic Space Forms and Orbifold Compactification in $M$ Theory, Proc. Sci. WC2004, 17 (2004).

[11] G. Moore and E. Witten, Self-duality, Ramond-Ramond fields, and $K$-theory, JHEP 0005, 032 (2000) [hep-th/9912279].

[12] D. S. Freed, Determinant line bundles revisted, Lecture Notes in Pure and Appl. Math. 184 187, Marcel Dekker, New York 1997.

[13] D. S. Freed, Dirac charge quantization and generalized differential cohomology, hep-th/0011220.

[14] J. Lott, $\mathbb{R} / \mathbb{Z}$-Index Theory, Comm. in Anal. and Geom. 2, 279 (1994).

[15] J. de Boer, R. Dijgraaf, K. Hori, A. Keurentjes, J. Morgan, D. R. Morrison, and S. Sethi, Triples, Fluxes, and Strings, Adv. Teor. Math. Phys. 4, 995 (2002) [hep-th/0103170].

[16] M. F. Atiyah, V. K. Patodi, and I. M. Singer, Spectral Asymmetry and Riemannian Geometry. I, Math. Proc. Camb. Phil. Soc. 77, 43 (1975).

[17] M. F. Atiyah, V. K. Patodi, and I. M. Singer, Spectral Asymmetry and Riemannian Geometry. II, Math. Proc. Camb. Phil. Soc. 78, 405 (1975)

[18] M. F. Atiyah, V. K. Patodi, and I. M. Singer, Spectral Asym- metry and Riemannian Geometry. III, Math. Proc. Camb. Phil. Soc. 79, 71 (1976)

[19] H. Moscovici and R. Stanton, Eta invariants of Dirac operators on locally symmetric manifolds, Invent. Math. 95, 629 (1989).

[20] M. F. Atiyah and F. Hirzebruch, Analytical Cycles on Complex Manifolds, Topology 1, 25 (1962).

[21] R. M. Switzer, Algebraic Topology: An Introduction, SpringerVerlag, 1978.

[22] R. M. G. Reis and R. J. Szabo, Geometric K-Homology of Flat D-Brtanes, hep-th/0507043.

[23] F. Quinn, Ends of Maps, II, Invet. Math. 68, 353 (1982).

[24] H. Bass, A. Heller, and R. Swan, The Whitehead group of a polynomial extension, Publ. Inst. Hautes Etudes Sci. 22, 61 (1964).

[25] J. Stallings, Whitehead torsion of free products, Ann. of Math. 82, 354 (1965).

[26] F. T. Farrell and L. E. Jones, Topological rigidity for compact nonpositively curved manifolds, Differential Geometry: Riemannian Geometry (Los Angeles, CA 1990), Proc. Sympos. Pure Math. 54, Part 3, American Mathematical Society, Providence, R. I. (1993) 229.

[27] B. Hu, Whitehead groups of finite polyhedra with non-positive curvature, J. Diff. Geom. 38, 501 (1993).

[28] F. Waldhausen, Algebraic $K$-theory of generalized free products, Parts 1 and 2, Ann. of Math. 108, 135 (1978).

[29] G. Higman, The units of group-rings, Proc. London Math. Soc. 46, 231 (1940).

[30] F. T. Farrell and L. E. Jones, Algebraic $K$-theory of spaces stratified fibered over hyperbolic orbifolds, Proc. Natl. Acad. Sci. USA, 83, 5364 (1986).

[31] F. T. Farrell and L. E. Jones, Isomorphism congectures in algebraic K-theory, J. Amer. Math. Soc. 6, 249 (1993).

[32] J. F. Davis and W. Lück, Spaces over a category and essembly maps in isomorphim congectures in $K$ - and L-theory, $\mathrm{K}$ theory 15, 201 (1998).

[33] E. Bercove, F. T. Farrell, D. J. Pineda, and K. Pearson, The Farrell-Jones isomorphism conjecture for finite covolume hyperbolic actions and the algebraic $K$-theory of Bianchi groups, Trans. Amer. Math. Soc. 352, 5689 (2000). 